\title{
ATENÇÃo PRIMÁRIA NO PRÉ E PÓS-PARTO EM MULHERES GRÁVIDAS EM UM BAIRRO DO NORDESTE DO BRASIL
}

\author{
Rosemary Araújo MONTEIRO ${ }^{1}$; Hugo Navarro Carlos CAVALCANTI ${ }^{2}$; Neila Alves de \\ QUEIROZ $^{3}$; Gil Dutra FURTADO ${ }^{4}$
}

\begin{abstract}
${ }^{1}$ Graduada em Fisioterapia/Universidade Federal da Paraíba (UFPB); Mestra em Desenvolvimento e Meio Ambiente (PRODEMA)/UFPB; Professora Adjunta IV/Universidade Federal do Rio Grande do Norte (UFRN), Brasil. E-mail: rosemarymonteiro1@gmail.com

${ }^{2}$ Graduando em Fisioterapia/UFRN; Monitor do Programa de Educação pelo Trabalho para a Saúde (PETSAÚDE)/GRADUASUS/UFRN, Brasil. E-mail: hugo_.navarro@outlook.com

${ }^{3}$ Graduação em Fisioterapia/UFRN; Fisioterapeuta/Núcleo de Apoio à Saúde da Família (NASF-RN), Brasil. Email: neilaaq@ hotmail.com

${ }^{4}$ Engenheiro Agrônomo/UFPB; Doutor em Psicobiologia/Universidade Federal do Rio Grande do Norte (UFRN); Agrônomo-Sócio da Cooperativa de Agronegócio (COOPAGRO), Brasil. E-mail: gdfurtado@hotmail.com
\end{abstract}

Resumo. No Brasil nascem várias crianças que requerem dos profissionais da área de saúde uma grande responsabilidade que é cuidar de cada gestante e de cada criança que nasce e começa a se desenvolver. Esta responsabilidade aumenta ainda mais diante do fato de que, sem a devida atenção, muitas dessas crianças morrem antes de completar um ano de idade. Cuidar da vida de cada gestante e de cada criança significa alguém que cuida. Para garantir um acompanhamento adequado durante o pré-natal e o controle no primeiro ano de vida foi previsto o fortalecimento das atividades entre o ensino, serviço e comunidade com o objetivo de oferecer uma assistência às gestantes e ao bebê. A disciplina SACI (Saúde e Cidadania) II elaborou atividades com as comunidades gestantes do Bairro "Nordeste", Natal/RN, junto com a colaboração e empenho de cada profissional da "USF Nordeste". Foram realizadas atividades com palestras temáticas em cuidados com a amamentação, alimentação saudável, exercícios funcionais adaptados a condição gestante, momento do parto e pós-parto, cuidados com o bebê, higiene do sono, vacinação e massagem "Shantala". Como resultado, uma cartilha foi elaborada, com uma linguagem simples e de forma lúdica, proporcionando às mulheres um meio rápido para tirar dúvidas e um guia para acompanhamentos com a equipe de saúde.

Palavras-chave: Cidade de Natal; Gestantes; Saúde e Cidadania.

\section{PRIMARY CARE FOR PRE AND POST-BIRTH IN PREGNANT WOMEN IN A NEIGHBORHOOD FROM NORTHEAST BRAZIL}

Abstract. In Brazil, several children are born who require of the professionals of the health area a
great responsibility that is to take care of each pregnant and of each child that is born and begins to 
develop. This responsibility is further heightened by the fact that, without proper attention, many of these children die before reaching one year of age. Taking care of the life of each pregnant and each child means someone who cares. To ensure adequate follow-up during prenatal care and control in the first year of life, it was planned to strengthen activities between teaching, service and community with the objective of offering assistance to pregnants and the baby. The SACI (Health and citizenship) II discipline developed activities with the pregnant communities of the "Nordeste" neighborhood, Natal/RN, along with the collaboration and commitment of each "USF Nordeste" professional. Activities were held with thematic lectures on breastfeeding care, healthy eating, functional exercises adapted to the pregnant condition, delivery and postpartum, baby care, sleep hygiene, vaccination and "Shantala" massage. As a result, a primer was developed with simple language and playfulness, providing women with a quick way of asking questions and a guide for follow-up with the health team.

Keywords: Natal city; Pregnants; Health and Citizenship.

\section{CUIDADO PRIMARIO PARA PRE Y POST-NACIMIENTO EN MUJERES EMBARAZADAS EN UN BARRIO DEL NORDESTE DE BRASIL}

Resumen. En Brasil, nacen varios niños que requieren de los profesionales del área de salud una gran responsabilidad que es cuidar de cada gestante y de cada niño que nace y comienza a desarrollarse. Esta responsabilidad aumenta aún más ante el hecho de que, sin la debida atención, muchos de estos niños mueren antes de cumplir un año de edad. Cuidar la vida de cada gestante y de cada niño significa alguien que cuida. Para garantizar un seguimiento adecuado durante el prenatal y el control en el primer año de vida se previó el fortalecimiento de las actividades entre la enseñanza, servicio y comunidad con el objetivo de ofrecer una asistencia a las gestantes y al bebé. La disciplina SACI (Salud y Ciudadanía) II elaboró actividades con las comunidades gestantes del Barrio "Nordeste", Natal/RN, junto con la colaboración y empeño de cada profesional de la "USF Nordeste". Se realizaron actividades con charlas temáticas en cuidados con la lactancia, alimentación sana, ejercicios funcionales adaptados a la condición gestante, momento del parto y posparto, cuidados con el bebé, higiene del sueño, vacunación y masaje "Shantala". Como resultado, una cartilla fue elaborada, con un lenguaje simple y de forma lúdica, proporcionando a las mujeres un medio rápido para sacar dudas y una guía para acompañamientos con el equipo de salud.

Palabras clave: Ciudad de Natal; Embarazadas; Salud y Ciudadanía.

\section{INTRODUÇÃO}

A saúde da mulher por muito tempo foi negligenciada, porém vem sendo criadas políticas públicas como Política Nacional de Atenção Integral à Saúde da Mulher - PNAISM (COSTA, 2012) que visa atender a mulher em todos os âmbitos da saúde. A rede cegonha a qual garante 
assistência durante planejamento reprodutivo e a atenção humanizada à gravidez, ao parto e ao puerpério, bem como assegura às crianças o direito ao nascimento seguro, crescimento e desenvolvimento saudável, oferecendo serviços como pré natal, parto, puerpério e atenção integral à saúde da criança (MINISTÉRIO DA SAÚDE, 2018). Essa abordagem está incluída na Política Nacional de Atenção Básica (PNAB), que é resultado da experiência acumulada por conjunto de atores envolvidos historicamente com o desenvolvimento e a consolidação do Sistema Único de Saúde (SUS), como movimentos sociais, usuários, trabalhadores e gestores das três esferas de governo (BRASIL, 2012).

Objetivou-se com este, promover a integração das mulheres nas fases gravídicopuerperal aos programas de assistência à saúde da mulher e criação de um vínculo com a Unidade de Saúde da Família (USF) do Bairro Nordeste, na cidade de Natal, estado do Rio Grande do Norte/Brasil, com a finalidade de permitir um melhor auxílio dos profissionais no acompanhamento das mesmas.

\section{MATERIAL E MÉTODOS}

Com o uso de metodologias ativas, realizamos várias atividades com o tema: "Saúde da mulher na gravidez, puerpério e cuidados com o bebê”. As ações consistiram em encontros realizados entre os alunos de diversos cursos das áreas de saúde da Universidade Federal do Rio Grande do Norte (UFRN), profissionais da USF Nordeste e mulheres gestantes residentes no Bairro. Foram planejados dois encontros como modo de interação. Na primeira reunião, utilizamos dinâmicas como: 1) "Mitos e Verdades", com perguntas de verdadeiro e falso, em relação ao cuidado materno e do bebê; 2) Distribuição de folhetos informativos e rodas de conversas com orientações gerais a respeito da saúde materna e do bebê; e 3) a divulgação de quais serviços da USF dispõe para atendê-las. No segundo momento: 1) Realizamos palestras expositivas e visuais sobre o momento do parto (posicionamentos adequados, massoterapia, exercícios do assoalho pélvico, padrões respiratórios e técnicas de relaxamento); 2) Técnica de massoterapia "Shantala", na qual foi usada de forma expositiva como meio de aproximação entre a mãe e seu filho. No final dos encontros, foram distribuídos brindes a cada participante.

\section{RESULTADOS}

Através das ações realizadas no Bairro Nordeste, foi possível trazer novas informações e esclarecer sobre os períodos da gravidez, parto e primeiros dias de vida do bebê. As mulheres participaram ativamente das dinâmicas, tirando suas dúvidas, construindo diálogos com outras 
mulheres presentes, além dos alunos e profissionais da unidade e ainda compartilhando experiências tanto próprias como de outras pessoas. Quando o conhecimento era novo para a maioria delas, as mesmas se mostraram abertas a aptas a colocar em prática. Foi possível reforçar a importância do acompanhamento periódico com a equipe de saúde da USF e todas mostraram bastante consciência dos benefícios desse auxílio disponível.

O contato proporcionou maior forma organizacional da equipe de saúde da família da USF Nordeste, com relação ao acolhimento, dados clínicos e acompanhamento do estado de saúde e bem-estar de cada mulher nos períodos do pré e pós-parto. Um compilado de tudo que foi abordado nas ações foi exemplificado por meio de uma cartilha (Fig. 1), com uma linguagem simples e de forma lúdica, proporcionando as mulheres um meio rápido para tirar algumas dúvidas e um guia para acompanhamentos com a equipe de saúde.

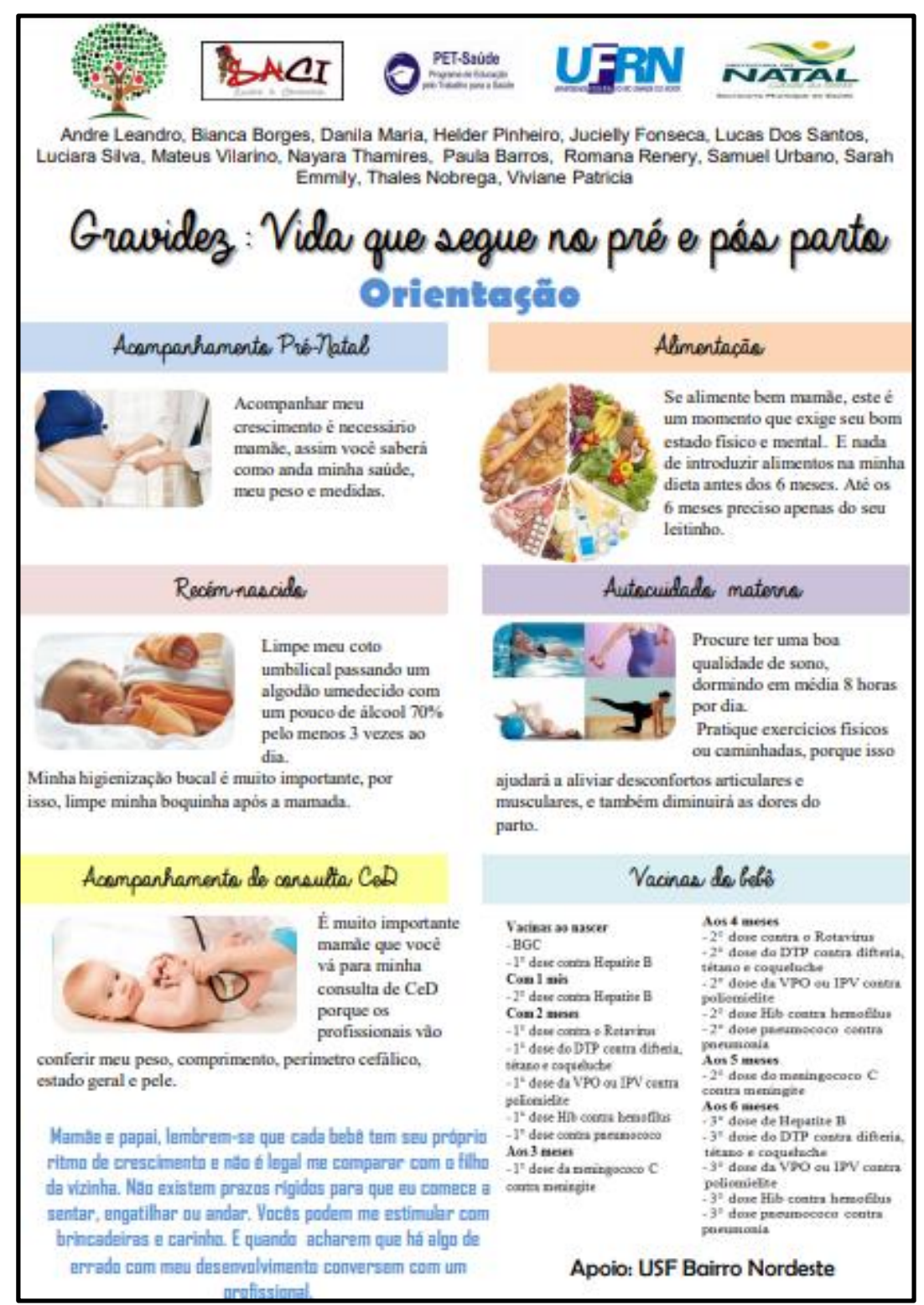


Figura 1. Cartilha resultante da atividade desenvolvida na Unidade de Saúde da Família.

Nesta perspectiva, a disciplina SACI II buscou mostrar aos alunos o modo de como as Políticas Públicas de Saúde funcionam, em especial a Estratégia de Saúde da Família, ensinado de forma prática como é organizada uma Unidade Básica de Saúde em todos os seus aspectos, juntamente com a interdisciplinaridade. Desta forma, proporciona aos alunos da área de saúde a possibilidade de vivenciar um pouco do que possivelmente será sua rotina de trabalho, bem como desenvolvendo o seu senso crítico a respeito da realidade da saúde pública brasileira. A disciplina também ensina o aluno a pensar em si mesmo como agente de transformação social e sua contribuição quanto pessoa e profissional na melhoria da qualidade de vida da sociedade como um todo.

\section{AGRADECIMENTOS}

Agradecemos ao apoio técnico fornecido pelos alunos da turma de alunos da SACI (Saúde \& Cidadania) 2 do Departamento de Saúde Coletiva da Universidade Federal do Rio Grande do Norte. Aos profissionais da Unidade de Saúde da Família do Bairro Nordeste/Natal - RN; PETSAUDE (Programa de Educação pelo Trabalho para a Saúde)/GRADUASUS.

\section{REFERÊNCIAS}

BRASIL. Ministério da Saúde. Política Nacional de Atenção Básica. Brasília, DF, 2012.

COSTA, A.M. Política de saúde integral da mulher e direitos sexuais e reprodutivos. In: GIOVANELLA, L.; ESCOREL, S.; LOBATO, L.V.C.; NORONHA, J.C.; CARVALHO, A.I. (Org.) Políticas e sistemas de saúde no Brasil. 2. ed. Rio de Janeiro: Fiocruz, 2012. p. 9791010 .

MINISTÉRIO DA SAÚDE/Departamento de Atenção à Saúde. Rede Cegonha. 2018. Disponível em: <http://dab.saude.gov.br/portaldab/ape_redecegonha.php>. Acesso em: 12 jul. 2018. 